\title{
Vacuum non-linear electrodynamic polarization effects in hard emission of pulsars and magnetars
}

\author{
V.I. Denisov, ${ }^{a}$ V.A.Sokolov, ${ }^{a, 1}$ S.I.Svertilov ${ }^{a}$ \\ ${ }^{a}$ Physics Department, Moscow State University, Moscow, Russia \\ E-mail: vid.msu@yandex.ru, sokolov.sev@inbox.ru, sis@coronas.ru
}

\begin{abstract}
The non-linear electrodynamics influence of pulsar magnetic field on the electromagnetic pulse polarization is discussed from the point of observation interpretation. The calculations of pulsar magnetic field impact on electromagnetic pulse polarization are made in such a way to make it easier to interpret these effects in space experiments. The law of hard emission pulse propagation in the pulsar magnetic field according to the vacuum nonlinear electrodynamics is obtained. It was shown, that due to the birefringence in the vacuum the front part of any hard emission pulse coming from a pulsar should be linearly polarized and the rest of pulse can have arbitrary polarization. Observational possibilities of vacuum birefringence are discussed. The estimations of detector parameters such as effective area, exposure time and necessity of polarization measurements with high accuracy are given. The combination of large area and extremely long exposure time gives the good opportunity to search the fine polarization effects like vacuum non-linear electrodynamics birefringence.
\end{abstract}

Keywords: gamma ray theory, X-rays, magnetic fields, X-ray pulsar

\footnotetext{
${ }^{1}$ Corresponding author.
} 


\section{Contents}

1 Introduction

2 The law of hard emission pulse propagation in the pulsar magnetic field according to the vacuum non-linear electrodynamics

3 Observational possibilities of vacuum nonlinear electrodynamics effects

4 Conclusions

\section{Introduction}

Although about a few hundreds of pulsars are discovered in hard X-rays and gamma-rays and their temporal and spectral parameters were studied with good accuracy, there are no sufficiently completed data about polarization properties of these astrophysical objects. A very limited number of space experiments, which were able to study polarization in hard emission, were realized at all. Mainly, there were observations of solar flares, such as polarization measurements at Soviet and Russian satellites InterCosmos [1] and Coronas-F [2] and RHESSI space observatory, which give the principle possibility of polarization detection of solar flare gamma-rays and cosmic gamma-ray bursts (GRB) [3]

Polarimetry is a powerful diagnostic of specific phenomena at work in cosmic sources in the radio-wave and optical energy bands, but very few results are available at high photon energies: the only significant observation in the X-gamma energy range, to date, is the measurement of a linear polarization fraction of $P=19+1 \%$ of the $2.6 \mathrm{keV}$ emission of the Crab nebula by a Bragg polarimeter on board OSO-8 [4].At higher energies, hard-X-ray and softgamma-ray telescopes that have flown to space in the past (for ex. COMPTEL CGRO, [5]) were not optimized for polarimetry, and their sensitivity to polarization was poor. Presently active missions INTEGRAL IBIS [6-8], and SPI [9, 10], have provided some improvement, with, in particular, mildly significant measurements of $P=28+6 \%$ (from 130 to $440 \mathrm{keV}$ ), [11] and $P=47_{-13}^{+19} \%$ (from 200 to $800 \mathrm{keV}$ ), [12] for the Crab Nebula.

A number of Compton polarimeter/telescope projects have been developed, some of which also propose to record photon conversions to $e^{+} e^{-}$pairs. A variety of technologies have been considered, such as scintillator arrays POGO [13], GRAPE [14], POLAR [15], Si or Ge microstrip detectors MEGA [16], ASTROGAM [17] or combinations of those ( $\mathrm{Si}+\mathrm{LaBr}_{3}$ for GRIPS [18], Si + CsI(Tl) for TIGRE [19], semiconductor pixel detectors CIPHER [20], liquid xenon LXeGRIT [21] and gas mixture CF4 at $3 \mathrm{~atm}$ [22]) time projection chambers (TPC).

It is supposed that hard emission of astrophysical sources contained strongly magnetized neutron stars should be more-less polarized. It could be caused by geometry of emitting areas, i.e. accretion disk (in the case of neutron star in double system) or magnetic field configuration as well as by highly anisotropy of primary electron beams. The other reason is connected with influence on $\mathrm{X}$ and gamma ray propagation of specific physical conditions in neutron star magnetosphere. 
However, there are the other kind of physical processes, which can lead to polarization effects. It means the influence of very strong magnetic fields on the $\mathrm{X}$ and gamma ray polarization. The critical value is so called Schwinger field $B_{q}=m^{2} c^{3} /(e \hbar)=4.41 \cdot 10^{13}$ $\mathrm{G}$, above which the non-linear electrodynamic effects in vacuum become significant. It is impossible to realize such fields in ground experiments, so the astrophysical observations are the only way to test non-linear electrodynamic models. The strong magnetic field is not so rare in the Universe.

Some gamma-ray pulsars are characterized by magnetic fields near $B_{q}$ value, but for so called magnetars it could be even higher and reach about $10^{15} \mathrm{G}$. In such objects in the vicinity of strongly magnetized neutron star there are favorable conditions for non-linear electrodynamic effects, in particular, vacuum birefringence [23-25]. This effect may have different manifestations. One is the dramatically increases the linear polarization of the thermal radiation of the isolated neutron stars [26], from a level of a few per cent up to even 100 per cent, depending on the viewing geometry and the surface emission mechanism. Currently, this vacuum non-linear electrodynamics prediction can be tested by measurement of a polarization degree of isolated neutron star thermal emission.

First observations [27] of optical polarization from a thermally emitting isolated neutron star RX J1856.5-3754 shown that a linear polarization degree of this star thermal emission is $16.43+5.26$ per cent.

It is arguing in [27] that, independently on how thermal photons are produced, such a high value of linear polarization in the signal is extremely unlikely to be reproduced by models in which QED vacuum polarization effects are not accounted for.

In this paper there are presented the results of calculations of other vacuum birefringence manifestation in high magnetic field, which could be studied only by polarization measurements of pulsed hard emission.

Observations just in hard $\mathrm{X}$ and gamma rays are preferable to avoid the mentioned above polarization effects caused by the pulsar magnetosphere plasma influence, which are more weak for high energy photons.

General covariance equations of non-linear electrodynamics in post-Maxwellian approximation $\left(|\mathbf{E}|>B_{q},|\mathbf{B}|>B_{q}\right)$ have the form:

$$
\begin{gathered}
\frac{1}{\sqrt{-g}} \frac{\partial}{\partial x^{n}}\left\{\sqrt{-g} Q^{m n}\right\}=-\frac{4 \pi}{c} j^{m}, \\
\frac{\partial F_{m n}}{\partial x^{k}}+\frac{\partial F_{n k}}{\partial x^{m}}+\frac{\partial F_{k m}}{\partial x^{n}}=0,
\end{gathered}
$$

where

$$
\left.Q^{m n}=\left[1+\xi\left(\eta_{1}-2 \eta_{2}\right) J_{2}\right)\right] F^{m n}+4 \xi \eta_{2} F^{m i} F_{i k} F^{k n}
$$

here notations were introduced, such as determinant of the metric tensor $g$ and $\xi=1 / B_{q}^{2}$.

The magnitude of the dimensionless post-Maxwellian parameters $\eta_{1}$ and $\eta_{2}$ depends on the choice of vacuum non-linear electrodynamic model. In the Heisenberg-Euler non-linear electrodynamics [28], which is direct consequence of quantum electrodynamics, parameters $\eta_{1}$ and $\eta_{2}$ have the values $\eta_{1}=\alpha /(45 \pi)=5.1 \cdot 10^{-5}, \eta_{2}=7 \alpha /(180 \pi)=9.0 \cdot 10^{-5}$; in the Born-Infeld theory [29] they are equal $\eta_{1}=\eta_{2}=a^{2} B_{q}^{2} / 4$, where to the constant $a^{2}$ only lower estimation is known $a^{2}>1.2 \cdot 10^{-32} G^{-2}$. Effects of vacuum polarization in strong electromagnetic fields were studied in [30], birefringence and photon splitting for uniform magnetic field were analyzed in [31]. 
Birefringence in vacuum and its influence on the emission spectrum and photon propagation in the neutron star magnetosphere were discussed in details in [32], where it was presented that vacuum effects determine polarization properties of normal modes of electromagnetic waves propagating in the vicinity of neutron star. It was also shown, that magnetic vacuum effects can change the emission spectral parameters that should lead to peculiarities in energy spectra of $\mathrm{X}$ and gamma ray pulsars [33, 34].

Non-linear electrodynamic effects caused by non-uniform and non-stationary magnetic field of a neutron star including beam bending in the dipole magnetic field, electron-positron pair production, photon splitting and modulation by the low-frequency electromagnetic wave scattering in magnetic field of inclined rotator are discussed in [35]. Quantum electrodynamic effect in accreting neutron stars, in particular, one and two photon Compton scattering in the strong magnetic field and its acting on the emitting processes [36-38] and vacuum polarization effects in the field of charged compact object were also studied [39].

Calculations and modeling of non-linear electrodynamic influence of pulsar magnetic field on the electromagnetic pulse polarization were made repeatedly in many particular cases and with the use of different coordinate systems [24, 25, 40, 41]. However, in these cases coordinates were chosen from the terms of convenience and simplicity of theoretical calculations. Often it was not appropriate from the point of observation interpretation. In this work we avoid this drawback and make calculations of pulsar magnetic field impact on electromagnetic pulse polarization in such a way to make it easier to interpret these effects in space experiments.

It is necessary to note, that pulsar also have a strong gravitational field, which is also can impact on electromagnetic emission pulses. Because gravitational field cans only bend the trajectories of electromagnetic beams [42, 43], and it is not change their polarization, we will neglect gravitational effects in this work.

\section{The law of hard emission pulse propagation in the pulsar magnetic field according to the vacuum non-linear electrodynamics}

Let us consider a pulsar with radius $R_{N}$, which magnetic dipole moment $\mathbf{M}$ rigidly connected with pulsar "body". Then we suppose that the initial point of Cartesian system is placed into the pulsar center. Let us directed mutually orthogonal axes $X, Y$ and $Z$ in such a way, that $Z$ axis will pass through the center of the Earth, and directions of $X, Y$ will be cleared later. Let us consider, that at some time $t_{0}$ the short $\mathrm{X}$ or gamma-ray burst with arbitrary polarization was occur in the point $\mathbf{r}_{s}=\left\{x_{s}, y_{s}, z_{s}\right\}$ of pulsar magnetosphere. This emission will propagate as pulses along different beams in different directions underwent non-linear electrodynamic impact from pulsar magnetic field. Let us consider that detector on a satellite is placed in the point $\mathbf{r}_{d}=\left\{x_{d}, y_{d}, z_{d}\right\}$ in the vicinity of the Earth, that allows measurements of hard emission pulse polarization.

We will calculate here the polarization state of pulse coming to detector. To study process, which is interesting for us, there is no any necessity to solve the system of non-linear differential equations in partial derivatives of the first order (1). As it was shown in [41], propagation of electromagnetic pulse in outer persistent electromagnetic field according to vacuum non-linear electrodynamics can be describes by equations for isotropic geodesic in an effective space-time with metric tensor $G_{p m}^{(1,2)}$ :

$$
\frac{d k^{i}}{d \sigma}+G_{(1,2)}^{i m}\left[\frac{\partial G_{m n}^{(1,2)}}{\partial x^{p}}-\frac{1}{2} \frac{\partial G_{n p}^{(1,2)}}{\partial x^{m}}\right] k^{n} k^{p}=0,
$$


where $\sigma$ is affine parameter, $x^{n}=\left\{x^{0}=c t, x^{1}=x, x^{2}=y, x^{3}=z\right\}, k^{n}=d x^{n} / d \sigma$ is isotropic four-dimensional wave vector:

$$
G_{m i}^{(1,2)} \frac{d x^{m}}{d \sigma} \frac{d x^{i}}{d \sigma}=0
$$

The metric tensor $G_{p m}^{(1,2)}$ in post Maxwellian approximation of vacuum non-linear electrodynamics (1) depends on the metric tensor $g_{i m}$ of pseudo Riemannian space-time, which is the solution of Einstein equations from the outer electromagnetic field tensor $F_{i p}$ and from the polarization of considering electromagnetic wave:

$$
\begin{aligned}
& G_{(1,2)}^{i m}=g^{m i}+4 \eta_{1,2} \xi F^{i n} F_{n \cdot}^{\cdot m}, \\
& G_{m n}^{(1,2)}=g_{m n}-4 \eta_{1,2} \xi F_{m k} F_{\cdot n}^{k .},
\end{aligned}
$$

where index "1" refers to the first linearly polarized normal mode, and index "2" is refers to the second normal mode, which has the orthogonal polarization relatively to the first one. The indexes of electromagnetic field tensor $F_{m k}$ in the relation (4) can be upped with the use of metric tensor of pseudo Riemannian space-time $g^{m i}$. The tensor components $G_{(1,2)}^{i m}$ and $G_{m n}^{(1,2)}$ with post Maxwellian accuracy are inversed to each other:

$$
G_{(1,2)}^{i m} G_{m n}^{(1,2)}=\delta_{n}^{i}+O\left(\xi^{2} F^{i p} F_{p m} F^{m k} F_{k n}\right),
$$

where $\delta_{n}^{i}$ is the Kronecker tensor.

Existence of two relations $G_{m n}^{(1)}$ and $G_{m n}^{(2)}$ or effective space-time metric tensor means that according to the vacuum non-linear electrodynamics electromagnetic wave birefringence occurs, i.e. at $\eta_{1} \neq \eta_{2}$ any electromagnetic wave splits onto the two normal modes with mutually orthogonal linear polarization, which pass in the outer field with non-equal velocities. As it was shown in [44] occurrence of effective space-time with metric tensor $G_{p m}^{(1,2)}$, does not mean that Einstein Principe of equivalence violets in the electrodynamics. Presence of second terms in expression (4) means that not only gravitational field described by the metric tensor $g_{n k}$, affects on the electromagnetic wave propagation, but outer electromagnetic field also.

Equations (2)-(3) easier to solve, choosing as independent variable coordinate $z$, but not the affine parameter $\sigma$. In this case we have from the equations (2):

$$
\begin{aligned}
\frac{d^{2} x^{0}}{d z^{2}} & =\left\{G_{(1,2)}^{3 m} \frac{d x^{0}}{d z}-G_{(1,2)}^{o m}\right\}\left\{\frac{\partial G_{n m}^{(1,2)}}{\partial x^{p}}-\frac{1}{2} \frac{\partial G_{n p}^{(1,2)}}{\partial x^{m}}\right\} \frac{d x^{n}}{d z} \frac{d x^{p}}{d z} \\
\frac{d^{2} x}{d z^{2}} & =\left\{G_{(1,2)}^{3 m} \frac{d x}{d z}-G_{(1,2)}^{1 m}\right\}\left\{\frac{\partial G_{n m}^{(1,2)}}{\partial x^{p}}-\frac{1}{2} \frac{\partial G_{n p}^{(1,2)}}{\partial x^{m}}\right\} \frac{d x^{n}}{d z} \frac{d x^{p}}{d z} \\
\frac{d^{2} y}{d z^{2}} & =\left\{G_{(1,2)}^{3 m} \frac{d y}{d z}-G_{(1,2)}^{2 m}\right\}\left\{\frac{\partial G_{n m}^{(1,2)}}{\partial x^{p}}-\frac{1}{2} \frac{\partial G_{n p}^{(1,2)}}{\partial x^{m}}\right\} \frac{d x^{n}}{d z} \frac{d x^{p}}{d z}
\end{aligned}
$$

Equation (3)in this case has the form:

$$
G_{m n}^{(1,2)} \frac{d x^{m}}{d z} \frac{d x^{n}}{d z}=0 .
$$

Because the relation (6)is the first integral of equation (5), the equation system (5)-(6) is linearly dependent. Thus, one of the equations of this system can be omitted. It is easier 
to choose the first equation of system (5) as such one. These equations allow us to find the equation for rays $x=x(z), y=y(z)$ and the law of electromagnetic pulse propagation along these rays $x^{0}=c t=x^{0}(z)$. Because for each normal mode we are interested in only one ray along which pulse passes from the point $\mathbf{r}=\mathbf{r}_{s}$ in the point $\mathbf{r}=\mathbf{r}_{d}$, let us âô require that relations

$$
\begin{gathered}
x^{0}\left(z_{s}\right)=c t_{0}, \quad x\left(z_{s}\right)=x_{s}, \\
y\left(z_{s}\right)=y_{s}, \quad x\left(z_{d}\right)=x_{d}, \quad y\left(z_{d}\right)=y_{d} .
\end{gathered}
$$

should valid as the initial conditions to equations (5)-(6). To solve equations (5) - (6) with post-Maxwellian accuracy it is enough to take in Maxwellian approximation the pulsar dipole magnetic field induction vector $\mathbf{B}$ components:

$$
\mathbf{B}=\frac{3(\mathbf{M} \mathbf{r}) \mathbf{r}-\mathbf{M} r^{2}}{r^{5}} .
$$

It means that non-zero components of pulsar magnetic field tensor in relation (4) are:

$$
F_{21}=-F_{12}=B_{z}, F_{13}=-F_{31}=B_{y}, F_{32}=-F_{23}=B_{x} .
$$

The non-zero components of pseudo-Riemannian space-time metric tensor $g_{i m}$ if to neglect the gravitational field take a form:

$$
g_{00}=1, g_{11}=g_{22}=g_{33}=-1 .
$$

If to substitute relations (8) - (9) into expressions (4), we find the explicit form of non-zero components of effective space-time metric tensor $G_{p m}^{(1,2)}$ :

$$
\begin{gathered}
G_{00}^{(1,2)}=1, \\
G_{\alpha \beta}^{(1,2)}=-\delta_{\alpha \beta}\left\{1+4 \xi \eta_{1,2}\left[\frac{3(\mathbf{M} \mathbf{~ r})^{2}}{r^{8}}+\frac{\mathbf{M}^{2}}{r^{6}}\right]\right\}+ \\
+4 \xi \eta_{1,2}\left[\frac{9(\mathbf{M} \mathbf{~ r})^{2}}{r^{10}} x_{\alpha} x_{\beta}+\frac{M_{\alpha} M_{\beta}}{r^{6}}-\frac{3(\mathbf{M} \mathbf{r})}{r^{8}}\left(M_{\alpha} x_{\beta}+x_{\alpha} M_{\beta}\right)\right],
\end{gathered}
$$

where $\alpha, \beta \ldots=1,2,3, x_{\alpha}=\{-x,-y,-z\}, M_{\alpha}=\left\{-M_{x},-M_{y},-M_{z}\right\}$.

The detailed solution of equations (5)-(6) with effective space-time metric tensor which are satisfied the initial conditions (7) is presented in Attachment A. Let us write the law of electromagnetic pulse passing $x^{0}=x^{0}(z)$ along the ray connecting the points $\mathbf{r}_{s}$ and $\mathbf{r}_{d}$ :

$$
t_{1,2}(z)=t_{0}+\frac{\left(z-z_{s}\right)}{c}+\eta_{1,2} \xi\left[\widetilde{t}(z)-\widetilde{t}\left(z_{s}\right)\right],
$$

where notation is introduced

$$
\begin{gathered}
\widetilde{t}(z)=\left[25\left(M_{x} x_{s}+M_{y} y_{s}\right)^{2}+q^{2}\left(16 \mathbf{M}^{2}-M_{z}^{2}\right)\right]\left[\frac{z\left(3 \rho^{2}+2 q^{2}\right)}{64 \rho^{4} q^{6}}+\frac{3}{64 q^{7}} \operatorname{atan}\left(\frac{z}{q}\right)\right]+\quad(A 13) \\
+\frac{1}{\rho^{6} q^{2}}\left[5 z\left(M_{x} x_{s}+M_{y} y_{s}\right)^{2}+16 q^{2} M_{z}\left(M_{x} x_{s}+M_{y} y_{s}\right)+3 M_{z}^{2} q^{2} z\right]+\frac{9}{4 \rho^{8}}\left[z\left(M_{x} x_{s}+M_{y} y_{s}\right)^{2}-\right. \\
\left.-q^{2} M_{z}\left(2 M_{x} x_{s}+2 M_{y} y_{s}+M_{z} z\right)\right] .
\end{gathered}
$$


To study pulsar magnetic field impact on hard emission pulse polarization let us assume that its pulse at the point of its appearance had arbitrary polarization or was not polarized. Due to the vacuum non-linear electrodynamics birefringence this pulse splits on two pulses with mutually orthogonal linear polarizations. These pulses propagate in the pulsar magnetic field with non-equal velocities. They have fronts, which coincided at the initial time. The front of faster mode comes to the detector before, than the front of slow mode on a time interval equal $\Delta t=\left|t_{2}\left(z_{d}\right)-t_{1}\left(z_{d}\right)\right|$. It means that during the time $\Delta t$ only fast normal mode of a pulse will pass though the detector and it will detect the linear polarization at this part of pulse.

After a time $\Delta t$ the front of other normal pulse mode comes to the detector. Superposition of these normal modes in time produces in the detector emission with arbitrary polarization. Hence, according to the vacuum non-linear electrodynamics the front part of any hard emission pulse with duration $\Delta t$ ) coming from a pulsar should be linearly polarized and the rest of pulse can have arbitrary polarization. Electromagnetic pulse of faster mode will pass though the detector before the pulse of slow mode. After that the trailing edge of this pulse passed through the detector during a time $\Delta t$ only the trailing edge of slow mode pulse with orthogonal polarization will be detected. Thus, the "tail" with duration $\Delta t$ of any hard emission pulse underwent the non-linear electrodynamics impact of pulsar magnetic field should be linearly polarized, and its polarization will be orthogonal to polarization of the pulse front.

Thus, to test the predictions of vacuum non-linear electrodynamics, it is necessary control the polarization state of hard emission pulses coming from magnetars and pulsars on all their duration. If to use the expressions (A3) and (A13), we can calculate the time $\Delta t=|\Delta \tau|$ :

$$
\begin{gathered}
\Delta \tau=t_{2}\left(z_{d}\right)-t_{1}\left(z_{d}\right)=\left(\eta_{2}-\eta_{1}\right) \xi\left\{\left[\frac{3 \pi}{128 q^{7}}-\frac{z_{s}\left(3 \rho^{2}+2 q^{2}\right)}{64 r_{s}^{4} q^{6}}-\frac{3}{64 q^{7}} \operatorname{atan}\left(\frac{z_{s}}{q}\right)\right] \times\right. \\
\times\left[25\left(M_{x} x_{s}+M_{y} y_{s}\right)^{2}+q^{2}\left(16 \mathbf{M}^{2}-M_{z}^{2}\right)\right]-\frac{1}{8 r_{s}^{6} q^{2}}\left[5 z_{s}\left(M_{x} x_{s}+M_{y} y_{s}\right)^{2}+\right. \\
\left.+16 q^{2} M_{z}\left(M_{x} x_{s}+M_{y} y_{s}\right)+3 M_{z}^{2} q^{2} z_{s}\right]-\frac{9}{4 r_{s}^{8}}\left[z_{s}\left(M_{x} x_{s}+M_{y} y_{s}\right)^{2}-q^{2} M_{z}\left(2 M_{x} x_{s}+2 M_{y} y_{s}+M_{z} z_{s}\right)\right],
\end{gathered}
$$$$
\text { where } r_{s}=\sqrt{x_{s}^{2}+y_{s}^{2}+z_{s}^{2}} \text {. }
$$

\section{Observational possibilities of vacuum nonlinear electrodynamics effects}

The time interval $\Delta t$, which characterize delay of signals carried by electromagnetic waves polarized normally to each other essentially depends on the difference of post-Maxwellian parameters $\eta_{1}-\eta_{2}$. Thus, it is different in various models of vacuum nonlinear electrodynamics. In particular, in the Heisenberg-Euler electrodynamics this delay time may be about 1 mcs for typical pulsar, while in the Born-Infeld theory it is strictly equal to zero.

From the experimental point of view the effect of delaying of electromagnetic signals emitted from the poles of rotating neutron star will be revealed differently in the case of slow varying and burst-like or pulsed emission. In the case of slow varying emission the time dependence of detected intensity on one polarization mode will be shifted relatively to the time dependence of orthogonal mode intensity. This delay time will depend on the angle $\beta$ between neutron star magnetic momentum vector and the radius-vector of the point of 
detector place. Lag effect will manifest itself in another way in the case of burst-like or pulsed emission (photon beam) which duration is higher than time $\Delta t$, defined by expression (11).

In this case, if arbitrary polarized pulses are emitted, the detected pulses will have the variable polarization along their length, i.e. at $\eta_{1}>\eta_{2}$ the pulse front part of duration $\tau=\Delta t$ should be polarized normally to the neutron star magnetic meridian plane and the other part of a pulse will be polarized randomly in general case. If we assume that neutron star angular velocity $\Omega$ is sufficiently small, then the linear velocity of points on the neutron star surface will be much less than light velocity in vacuum, and electromagnetic signal propagation time in the area $r \sim 5 R_{s}$ of strong magnetic field, where the non-linear electrodynamic and gravitational actions on these signals are most significant, will be much less than the star rotation period. Thus, the area of non-linear electromagnetic action will be in the near field of magneto-dipole emission, it was shown [45] that the lag time of signals with two main polarizations will be modulated due to the star rotation. It is well-known that time profiles of pulsar $\mathrm{X}$ - and gamma ray emission look like a sequence of pulses with period equal to the neutron star rotation period and pulse duration is determined mainly by emission beam width.

In the case of very narrow beam the pulsar emission time profile can be presented as periodic pulses, which duration much less than period. At $\eta_{1}>\eta_{2}$ obviously, that pulse front will be polarized linearly, which plane is normal to the neutron star magnetic meridian. As it following from (11) duration of this polarized pulse front will change from zero to maximal value according to (11). Because the pulse duration $\tau$ is less than period $2 \pi / \Omega$, periodic change of intensity for time $\tau$ will not significant, thus for a real analysis it is possible to assume that polarized part of a pulse is constant. Its value will depend on observation line direction relatively to the neutron star meridian plane.

It was noted above that vacuum non-linear electrodynamic effects caused by the acting of very strong fields (including birefringence) are the same as non-linear effects in a matter. In this case strong magnetic field can be considered as effective matter. Thus, emission parameters determined by vacuum non-linear electrodynamic effects can be masked principally by non-linear influence of a matter. In particular, due to the Faraday effect, non-polarized emission scattered on plasma electrons becomes partially linearly polarized due to the inhomogeneous distribution of polarization plane rotation angles.

Such effect was analyzed for the cases of light propagation in magnetized stellar wind [46], strongly magnetized optically thick accretion disk [47], magnetized cone shell, which can be considered as model of relativistic jet [48]. However, non-linear electrodynamic effects caused by the matter influence will dominate on vacuum effects mainly for photons from long wave bands of electromagnetic spectrum, i.e. optics and radio. Indeed, polarization properties of matter are determined significantly by dielectric constant $\epsilon$, for which the expression [49] is well-known:

$$
\varepsilon=1-\frac{4 \pi N_{e} \hbar^{2} e^{2}}{m_{e} E_{\gamma}^{2}},
$$

where $E_{\gamma}$ is the photon energy, $N_{e}$ is the electron concentration in plasma, $m_{e}$ is the electron mass, h is the Plank constant. If to substitute in eq. (12) the typical $N_{e} \sim 10^{19} \mathrm{~cm}^{3}$ then we obtain for $E_{\gamma}=0.1 \mathrm{MeV} \varepsilon=1-10^{-11}$.

Thus, the matter influence on polarization properties of $\mathrm{X}$ ray and gamma emission is pitifully. This conclusion is confirmed by digital calculations, which shows that polarization degree of emission after it passed through magnetic plasma decreases with decreasing of its wave length [50]. 
Vacuum non-linear effects also can be connected with some exotic processes, for example, with mutual transformation in magnetic field of photons and light Goldstone bosons (axions). Electromagnetic emission occurred from the axion decay in magnetic field can be highly polarized [51]. However, according to [51] axion input in dielectric constant is inversely proportional to the square of the photon frequency (or energy) (compare with (12)). Thus, such processes can't be significant for determination of polarization properties of high energy photons.

So, it could be expected that revealing of vacuum non-linear electrodynamic effects will be most pure just in $\mathrm{X}$ and gamma rays from pulsars. It was shown above, that non-linear electrodynamics birefringence of $\mathrm{X}$ and gamma rays in strong magnetic field of pulsars and magnetars can be revealed as about 1 mcs lag of two signal modes almost $100 \%$ polarized in mutually normal directions. To measure such lag experimentally, it is necessary to realize accurate polarization measurements allowing obtain the mean pulsation curve for linearly polarized and non-polarized pulse components with fine time resolution (about $0.1 \mathrm{mcs}$ ). It is also necessary to measure with high accuracy $(\sim 0.1 \mathrm{mcs})$ the arrival time of detected quanta during the all-time of observations.

To make a final choice in favor of one or another experimental method, let us analyze the main factors that determine sensitivity of polarization measurements. For example, consider the Compton scattering technique as the most universal method of polarization measurements in hard X ray and gamma ray astronomy. Let $I$ is the total number of signal counts, $N$ is the background (noise) counts. Then for expected signal to noise ratio expressed in number of standard deviation $\sigma$ for given exposure time we have for polarization measurements an expression:

$$
\sigma=\frac{\mu I P}{\sqrt{2(I+N)}},
$$

where $P$ is the polarization degree and $\mu$ is the instrumental polarization factor, in which source position on the sky is also taken into account.

From (13) it is possible to obtain the estimation of minimally detectable polarization degree $P_{\min }$.

As it following from (13), to increase sensitivity it is necessary to minimize nose, increase the useful signal and increase the instrumental polarization factor $\mu$. The number of useful signal counts depends on the detected flux intensity $J$, which is expressed in $\mathrm{cm}^{-2} s^{-1}$ and equal to

$$
I=J S_{(e f f)} \Delta t
$$

where $S_{\text {eff }}$ is the effective detector area, $\delta t$ is the time set of signal statistics, which in the case of burst or pulse like signal is equal to the burst (pulse) duration and in the case of slow varying signals is determined by the exposure time.

Thus, it is following from (14) that to increase the useful signal it is necessary maximally increase the detector effective area and the exposure time. Thus, the increasing of detector area is inevitably linked with increasing of its mass and sizes, it is necessary to estimate the reasonable limits, which are defined of course by the source observable luminosity.

To estimate conditions for detection of $100 \%$ polarization, i.e. $P_{\min }=1$, let us put $\mu=1$ and neglect noise. Then to provide the $3 \sigma$ significance level no less than about 25 counts should be detected at each time interval (bin), on which polarization is measured. To reveal the lag in about $1 \mathrm{mcs}$ time bin should be not more than $1 \mathrm{mcs}$ also at least.

Then in the case of continuous signal from pulsar no less than $25 T /(2(1 \mathrm{mcs}))$ counts should be accumulated for pulsation period $T$. For simplicity we assume that pulsation profile 
Table 1. Signal to noise ratio of polarized fraction of pulsar emission in different energy range for different background models.

\begin{tabular}{lcccr}
\hline & Energy & ranges, & $\mathrm{MeV}$ & \\
\hline SNR & $0.002-0.001$ & $0.05-0.1$ & $0.5-1.0$ & $100-500$ \\
\hline$\frac{I}{N_{\text {diff }}}$ & 1 & 6 & 150 & 250 \\
\hline$\frac{I}{\left(N_{\text {diff } f}+N_{\text {int }}\right)}$ & 1 & 5 & 6 & 0.25 \\
\hline
\end{tabular}

is as rectangular with pulse phase duration equal to the pulsation period $T$. If the intensity of signal pulsed component is $J$ (in cm-2s-1), then in the case of detector with effective area $S_{\text {eff }}$ for one pulsation period $J \odot T \odot S_{\text {eff }} / 2$ counts will be detected. Then to satisfy the condition of $100 \%$ polarization detection with 1 mcs time resolution the following expression should be valid:

$$
\frac{25 T}{(1 m c s)}=J S_{e f f} T n,
$$

where $n$ is the number of pulsation periods for a total exposure time.

Equality (15) can be rewritten as

$$
\frac{25 T}{(1 m c s)}=J S_{e f f} \Delta t
$$

where $\Delta t$ is the total exposure time of a source.

It is well-known, that Crab pulsar is the most intensive in different energy bands among the other pulsars. Its spectrum can be good approximated in wide energy range by power law with power index $\sim 2[52,53]$, Then for the Crab pulsar intensity the following estimations can be obtained for different energies: $J(E=20-100 \mathrm{keV})=4.610^{-2} \mathrm{phot} / \mathrm{cm}^{2} / \mathrm{s}, J(E=$ $0.1-1.0 \mathrm{MeV})=1.210^{-2} \mathrm{phot} / \mathrm{cm}^{2} / \mathrm{s}$. Taking into account that Crab pulsation period is equal $33 \mathrm{~ms}$, we obtain from (15) the estimations of factor $S_{e f f} \Delta t$.

For energy range $20-100 \mathrm{keV}$ effect can be detected in the case of 1 Crab intensity, if $S_{\text {eff }}=10^{3} \mathrm{~cm}^{2}, \Delta t \sim 100 \mathrm{ks}$ and for $1 \mathrm{mCrab}$ intensity, if $S_{\text {eff }}=10^{4} \mathrm{~cm}^{2}, \Delta t \sim 3 \mathrm{Ms}$. For energy range $0.1-1.0 \mathrm{MeV}$ we have respectively if $S_{\text {eff }}=10^{4} \mathrm{~cm}^{2}, \Delta t \sim 100 \mathrm{ks}$ for 1 crab and if $S_{\text {eff }}=10^{4} \mathrm{~cm}^{2}, \Delta t \sim 10 \mathrm{Ms}$ for $1 \mathrm{mCrab}$ It is necessary to note, that effective area is determined not only by detector geometry area, but also by efficiency of scattering or any other process, which is used for polarization measurements. For the all well-known polarization techniques, including Compton polarimeters, efficiency no more than $10 \%$, really it is about a few percent. Thus, the polarimeter geometry area should be taken at least on order more than obtained above estimations of its effective area.

To choose the optimal observational method, it is necessary to analyze the main background factors in different energy ranges. Generally, detector background counts $N$ can be presented as a sum of parts caused by natural or Galactic and Meta-galactic diffuse background $N_{\text {diff }}$ and intrinsic background of detector and satellite $N_{\text {int }}$. Possible signal to noise ratio (SNR) can be estimated from pulsar energy spectrum, which can be taken typically as Crab-like. The corresponding SNR values are presented in the Table 1 in relative units, in which SNR in the $2-20 \mathrm{keV}$ range is taken equal to 1 . These values are obtained for a instrument with FOV $2 \pi$ sr, i.e. intensities of all background components were multiplied on $2 \pi$. As it follows from the Table 1, if take into account only the natural background, the best SNR value is obtained for the ranges of soft (0.1-1.0 MeV) and high energy (0.1-0.5 GeV) 
gamma rays. However, it is necessary to note, that at the energies less than $0.1 \mathrm{MeV}$ MetaGalactic diffuse background is dominating and its input in detector counts is proportional to the instrument FOV. While at energies $>0.1 \mathrm{MeV}$ noise counts are determined mainly by spacecraft and detector intrinsic background and weakly depends on FOV.

The SNR values, in which contribution of intrinsic background was taken into account, are also presented in the Table 1. In this case, SNR has favorable values for the range of hard $\mathrm{X}$ rays and soft gamma rays. It can be explained by that energy spectrum of intrinsic background is extremely hard, (see, for ex. [54] ) in presentation $E^{J}$ it can even grow with energy. Of course intrinsic background depends strongly on the spacecraft mass, which should be as low as possible, but such energy dependence of intrinsic background intensity is valid for satellites.

\section{Conclusions}

Thus, we can conclude, that the optimal energy range for vacuum non-linear electrodynamics effect search is the range $0.05-1.0 \mathrm{MeV}$, which can be extended up to about $10 \mathrm{MeV}$. What about the factor $S_{\text {eff }} \Delta t$, it should be understood, that increasing of the instrument geometry area can't be infinite due to the limited resources of space experiments. Obviously, that detector area about $10^{4} \mathrm{~cm}^{2}$ is near that limit, which can be still realized in space experiment. Concerning the exposure time, it can be made about of all time of experiment, which can be about of years, in the case of constant orientation of the instrument on a source or in the case of monitor observations with wide FOV telescope.

Thus, just the long time monitor observations are necessary for the search and revealing of non-linear electrodynamics effects. In view of discussed above features it seems that use of monitor instruments based on the Compton polarimeters are the most realistic way to realize the vacuum non-linear electrodynamics birefringence search and observation. Compton polarimeters have a real advantage in view of SNR optimization, because they based on detection of pair coincidences of incident and scattered photons, that allows eliminate instrument background effectively. Besides, such instruments give an opportunity to locate the source of detected gamma quanta by revealing of useful signals from the background cause by spacecraft and detector intrinsic noise and the atmosphere gamma rays (in the case of near-Earth observations). By this, the most dangerous are random coincidences in limits of trigger time $\tau$ of those noise events, which can be detected independently in the diffuse and detector of scattered quanta. As it is well-known, if noise count $N$ is constant and equal in diffuser and detector, the count of random coincidences $M$ is equal $M=N^{\tau}$. Thus, value $M$ can be made negligible in the case of very small time window $\tau$, that is in accordance with discussed above time resolution about 0.1 mcs.

Because in the Compton process quanta scatter presumably at $90^{\circ}$ relative to the incidence direction and in the case of linear polarization they are scattered normally to the polarization plane, intensity of scattered quanta will be modulated harmonically on Azimuth angle. Measurements by Compton polarimeters mean the obtaining of histogram of coincidence pair distribution on Azimuth angle and its approximation by harmonic function. Then this function amplitude can be used as the measure of polarization degree. The main factor worsening the instrument polarization capabilities is Coulomb scattering, which decreases exponentially the instrument polarization factor and minimal detectable polarization degree respectively. For example, CGRO EGRET instrument intended for observations in the range 
of high energy gamma rays scatter factor decrease effectively polarization factor in $10^{-4}$ times that made this experiment non-sensitive to polarization measurements.

To increase the efficiency of polarimetry measurements it is necessary to maximize the number of pair coincidences corresponding to interactions in two neighbor detector pixels. For this the radius of detector pixel should be about one mass absorption length $\lambda$. In the case of most popular for gamma quantum detection dense crystals as semiconductor as CdZnTe for example, or scintillator as $\mathrm{LaBr}_{3}, \mathrm{CeBr}_{3}$ and Ce:GAGG the pixel radius should be choose about $0.5-1.0 \mathrm{~cm}$. Thus, the Compton polarimeter instrument which is useful for vacuum non-linear electrodynamics birefringence observations should be based on large area $\left(\sim 10^{4}\right.$ $\mathrm{cm}^{2}$ ) detector consists from small pixels of about $1 \mathrm{~cm}$ size.

It should be taken into account, that in most Compton telescopes the reconstruction of the direction of the incident photon provides an uncertainty area which has the shape of a thin cone arc. However, there is an alternative way to use a gas TPC, that is also called Electron Tracking Compton Camera (ETCC), which provides the tracking of the recoil electron from the first Compton interaction with a measurement of the direction of the recoil momentum. It allows to decrease the length of the arc and therefore to improve dramatically the sensitivity of the detector [22], and references therein).

Some of these telescopes are sensitive to photon energies up to tens of $\mathrm{MeV}$ in the Compton mode, but their sensitivity to polarisation above a few $\mathrm{MeV}$ is either nonexistent or undocumented. Wide-field gamma ray $(0.02-3.0 \mathrm{MeV})$ telescope Gammascope, which is elaborated now at SINP MSU in the frame of Russian space program is able to realize the polarization measurements based on Compton technique. This instrument is the position sensitive detector (PSD) with coding mask of quasi-spherical (dodecahedron) configuration. It FOV of about $2 \pi$ sr provides the continuous observation of a half of the sky during space experiment.

The instrument should consist from six PSD modules placed on the bottom parts of dodecahedron frame and from six coding mask panels from Wf or Ta placed on the top parts of dodecahedron frame, i.e. the each PSD module has an opposite coding mask panel and all system observes half of sky. Due to such configuration the exposure time of each source in the instrument FOV will be about the all-time of experiment, i.e. few years. It is supposed that PSD should consists from a large number of cylindrical scintillator $\left(\mathrm{CeBr}_{3}\right.$ or Ce:GAGG) pixels of about $0.5 \mathrm{~cm}$ diameter and $2 \mathrm{~cm}$ height. The dense scintillator like $\mathrm{CsI}(\mathrm{Tl})$ or BGO will be used as active shield. The total area in the compact configuration should be $\sim 10^{3}$ $\mathrm{cm}^{2}$ and $\sim 10^{4} \mathrm{~cm}^{2}$ in the optimal case.

The special mode of double coincidences is foreseen for polarization measurements. The combination of large area and extremely long exposure time gives the good opportunity to search the fine polarization effects like vacuum non-linear electrodynamics birefringence.

\section{A Detailed calculations}

Let us start the solving of the equation system (5)-(6) with initial conditions (7). With the use of relation (10), we then obtain for the first integral (6):

$$
\begin{gathered}
c^{2}\left(\frac{d t}{d z}\right)^{2}-\left(\frac{d \mathbf{r}}{d z}\right)^{2}\left\{1+4 \xi \eta_{1,2}\left[\frac{3(\mathbf{M} \mathbf{r})^{2}}{r^{8}}+\frac{\mathbf{M}^{2}}{r^{6}}\right]\right\}+ \\
+\frac{4 \xi \eta_{1,2}}{r^{6}}\left[\frac{3(\mathbf{M} \mathbf{r})}{r^{2}}\left(\mathbf{r} \frac{d \mathbf{r}}{d z}\right)-\left(\mathbf{M} \frac{d \mathbf{r}}{d z}\right)\right]^{2}=0 .
\end{gathered}
$$


Substituting (10) into the last two equations of system (5), we obtain:

$$
\begin{aligned}
& \frac{d^{2} x}{d z^{2}}=\eta_{1,2} \xi\left\{\left[\frac{180}{r^{12}}(\mathbf{M} \mathbf{r})^{2} x^{2} z+\frac{12}{r^{10}}(\mathbf{M} \mathbf{r})\left[9(\mathbf{M} \mathbf{r}) z-z\left(14 x M_{x}+8 y M_{y}\right)+\right.\right.\right. \\
& \left.\left.\left(3 x^{2}+8 y^{2}\right) M_{z}\right]+\frac{12}{r^{8}}\left[z\left(\mathbf{M}^{2}+M_{z}^{2}\right)-9(\mathbf{M} \mathbf{r}) M_{z}+\left(3 x M_{x}+y M_{y}\right) M_{z}\right]\right]\left(\frac{d x}{d z}\right)^{3}+\left[\frac{360}{r^{12}}(\mathbf{M} \mathbf{r})^{2} x y z-\right. \\
& \left.-\frac{24}{r^{10}}(\mathbf{M} \mathbf{r})\left[3 z\left(y M_{x}+x M_{y}\right)+5 x y M_{z}\right]+\frac{24}{r^{8}} M_{z}\left[x M_{y}+y M_{x}\right]\right]\left(\frac{d x}{d z}\right)^{2}\left(\frac{d y}{d z}\right)+ \\
& +\left[\frac{180}{r^{12}}(\mathbf{M} \mathbf{r})^{2} y^{2} z+\frac{12}{r^{10}}(\mathbf{M} \mathbf{r})\left[9(\mathbf{M} \mathbf{r}) z+\left(8 x^{2}+3 y^{2}\right) M_{z}-z\left(8 x M_{x}+14 y M_{y}\right)\right]+\right. \\
& \left.+\frac{12}{r^{8}}\left[z\left(\mathbf{M}^{2}+M_{z}^{2}\right)+\left(x M_{x}+3 y M_{y}\right) M_{z}-9(\mathbf{M} \mathbf{r}) M_{z}\right]\right]\left(\frac{d x}{d z}\right)\left(\frac{d y}{d z}\right)^{2}+ \\
& +\left[\frac{24}{r^{10}}(\mathbf{M} \mathbf{r})\left[15(\mathbf{M} \mathbf{r}) y+3\left(2 x^{2}+y^{2}\right) M_{y}+8 y\left(x M_{x}-z M_{z}\right)\right]-\frac{360}{r^{12}}(\mathbf{M} \mathbf{r})^{2} y\left(2 x^{2}+\right.\right. \\
& \left.\left.+y^{2}\right)+\frac{24}{r^{8}}\left[\left(z M_{z}-x M_{x}\right) M_{y}-3(\mathbf{M} \mathbf{r}) M_{y}+y\left(M_{z}^{2}-M_{x}^{2}\right)\right]\right]\left(\frac{d x}{d z}\right)\left(\frac{d y}{d z}\right)+ \\
& +\left[\frac{12}{r^{10}}(\mathbf{M} \mathbf{r})\left[21(\mathbf{M} \mathbf{r}) x+25 x^{2} M_{x}+8 x\left(y M_{y}-z M_{z}\right)+6 y^{2} M_{x}\right]-\right. \\
& -\frac{180}{r^{12}}(\mathbf{M} \mathbf{r})^{2} x\left(3 x^{2}+2 y^{2}\right)-\frac{12}{r^{8}}\left[5(\mathbf{M} \mathbf{r}) M_{x}+x\left(4 M_{x}^{2}+M_{y}^{2}-M_{z}^{2}\right)+\right. \\
& \left.\left.+\left(y M_{y}-z M_{z}\right) M_{x}\right]\right]\left(\frac{d x}{d z}\right)^{2}+\left[\frac { 1 2 } { r ^ { 1 0 } } ( \mathbf { M } \mathbf { r } ) \left[\left(8 x^{2}+5 y^{2}\right) M_{x}-9(\mathbf{M} \mathbf{r}) x+x\left(14 y M_{y}+\right.\right.\right. \\
& \left.\left.+8 z M_{z}\right)\right]-\frac{180}{r^{12}}(\mathbf{M} \mathbf{r})^{2} x y^{2}+\frac{12}{r^{8}}\left[(\mathbf{M} \mathbf{r}) M_{x}-x\left(2 M_{x}^{2}+M_{y}^{2}+M_{z}^{2}\right)-\right. \\
& \left.\left.-\left(3 y M_{y}+z M_{z}\right) M_{x}\right]\right]\left(\frac{d y}{d z}\right)^{2}+\left[\frac { 1 2 } { r ^ { 1 0 } } ( \mathbf { M } \mathbf { r } ) \left[24 z(\mathbf{M} \mathbf{r})+\left(25 x^{2}+19 y^{2}\right) M_{z}+\right.\right. \\
& \left.+8 z\left(x M_{x}-y M_{y}\right)\right]-\frac{180}{r^{12}}(\mathbf{M} \mathbf{r})^{2} z\left(3 x^{2}+y^{2}\right)-\frac{12}{r^{8}}\left[20(\mathbf{M} \mathbf{r}) M_{z}+\left(x M_{x}-y M_{y}\right) M_{z}+\right. \\
& \left.\left.+z\left(M_{x}^{2}-M_{y}^{2}-4 M_{z}^{2}\right)\right]\right]\left(\frac{d x}{d z}\right)+\left[\frac{24}{r^{10}}(\mathbf{M} \mathbf{r})\left[3 x y M_{z}+3 x z M_{y}+5 y z M_{x}\right]-\right. \\
& \left.-\frac{360}{r^{12}}(\mathbf{M} \mathbf{r})^{2} x y z-\frac{24}{r^{8}}\left(y M_{z}+z M_{y}\right) M_{x}\right]\left(\frac{d y}{d z}\right)+\frac{180}{r^{12}}(\mathbf{M} \mathbf{r})^{2} x\left(x^{2}+y^{2}\right)+ \\
& +\frac{12}{r^{10}}(\mathbf{M} \mathbf{r})\left[\left(3 x^{2}-5 y^{2}\right) M_{x}-24(\mathbf{M} \mathbf{r}) x+x\left(8 y M_{y}+14 z M_{z}\right)\right]+ \\
& \left.+\frac{12}{r^{8}}\left[6(\mathbf{M ~ r}) M_{x}-x\left(2 M_{x}^{2}+M_{y}^{2}+M_{z}^{2}\right)-\left(y M_{y}+3 z M_{z}\right) M_{x}\right]\right\}, \\
& \frac{d^{2} y}{d z^{2}}=\eta_{1,2} \xi\left\{\left[\frac{180}{r^{12}}(\mathbf{M} \mathbf{r})^{2} y^{2} z+\frac{12}{r^{10}}(\mathbf{M} \mathbf{r})\left[9(\mathbf{M} \mathbf{r}) z-z\left(14 y M_{y}+8 x M_{x}\right)+\left(3 y^{2}+8 x^{2}\right) M_{z}\right]+\right.\right. \\
& \left.+\frac{12}{r^{8}}\left[z\left(\mathbf{M}^{2}+M_{z}^{2}\right)-9(\mathbf{M} \mathbf{r}) M_{z}+\left(3 y M_{y}+x M_{x}\right) M_{z}\right]\right]\left(\frac{d y}{d z}\right)^{3}+\left[\frac{360}{r^{12}}(\mathbf{M} \mathbf{r})^{2} x y z-\right.
\end{aligned}
$$




$$
\begin{aligned}
& \left.-\frac{24}{r^{10}}(\mathbf{M} \mathbf{r})\left[3 z\left(x M_{y}+y M_{x}\right)+5 x y M_{z}\right]+\frac{24}{r^{8}} M_{z}\left[x M_{y}+y M_{x}\right]\right]\left(\frac{d y}{d z}\right)^{2}\left(\frac{d x}{d z}\right)+ \\
& +\left[\frac{180}{r^{12}}(\mathbf{M} \mathbf{r})^{2} x^{2} z+\frac{12}{r^{10}}(\mathbf{M} \mathbf{r})\left[9(\mathbf{M} \mathbf{r}) z+\left(8 y^{2}+3 x^{2}\right) M_{z}-z\left(8 y M_{y}+14 x M_{x}\right)\right]+\right. \\
& \left.+\frac{12}{r^{8}}\left[z\left(\mathbf{M}^{2}+M_{z}^{2}\right)+\left(y M_{y}+3 x M_{x}\right) M_{z}-9(\mathbf{M} \mathbf{r}) M_{z}\right]\right]\left(\frac{d y}{d z}\right)\left(\frac{d x}{d z}\right)^{2}+ \\
& +\left[\frac{24}{r^{10}}(\mathbf{M} \mathbf{r})\left[15(\mathbf{M} \mathbf{r}) x+3\left(2 y^{2}+x^{2}\right) M_{x}+8 x\left(y M_{y}-z M_{z}\right)\right]-\frac{360}{r^{12}}(\mathbf{M} \mathbf{~ r})^{2} x\left(2 y^{2}+\right.\right. \\
& \left.\left.+x^{2}\right)+\frac{24}{r^{8}}\left[\left(z M_{z}-y M_{y}\right) M_{x}-3(\mathbf{M} \mathbf{r}) M_{x}+x\left(M_{z}^{2}-M_{y}^{2}\right)\right]\right]\left(\frac{d y}{d z}\right)\left(\frac{d x}{d z}\right)+ \\
& +\left[\frac{12}{r^{10}}(\mathbf{M} \mathbf{r})\left[21(\mathbf{M} \mathbf{r}) y+25 y^{2} M_{y}+8 y\left(x M_{x}-z M_{z}\right)+6 x^{2} M_{y}\right]-\right. \\
& -\frac{180}{r^{12}}(\mathbf{M} \mathbf{r})^{2} y\left(3 y^{2}+2 x^{2}\right)-\frac{12}{r^{8}}\left[5(\mathbf{M} \mathbf{r}) M_{y}+y\left(4 M_{y}^{2}+M_{x}^{2}-M_{z}^{2}\right)+\right. \\
& \left.\left.+\left(x M_{x}-z M_{z}\right) M_{y}\right]\right]\left(\frac{d y}{d z}\right)^{2}+\left[\frac { 1 2 } { r ^ { 1 0 } } ( \mathbf { M } \mathbf { r } ) \left[\left(8 y^{2}+5 x^{2}\right) M_{y}-9(\mathbf{M} \mathbf{r}) y+y\left(14 x M_{x}+\right.\right.\right. \\
& \left.\left.+8 z M_{z}\right)\right]-\frac{180}{r^{12}}(\mathbf{M} \mathbf{r})^{2} y x^{2}+\frac{12}{r^{8}}\left[(\mathbf{M} \mathbf{r}) M_{y}-y\left(2 M_{y}^{2}+M_{x}^{2}+M_{z}^{2}\right)-\right. \\
& \left.\left.-\left(3 x M_{x}+z M_{z}\right) M_{y}\right]\right]\left(\frac{d x}{d z}\right)^{2}+\left[\frac { 1 2 } { r ^ { 1 0 } } ( \mathbf { M } \mathbf { r } ) \left[24 z(\mathbf{M} \mathbf{r})+\left(25 y^{2}+19 x^{2}\right) M_{z}+\right.\right. \\
& \left.+8 z\left(y M_{y}-x M_{x}\right)\right]-\frac{180}{r^{12}}(\mathbf{M} \mathbf{r})^{2} z\left(3 y^{2}+x^{2}\right)-\frac{12}{r^{8}}\left[20(\mathbf{M} \mathbf{r}) M_{z}+\left(y M_{y}-x M_{x}\right) M_{z}+\right. \\
& \left.\left.+z\left(M_{y}^{2}-M_{x}^{2}-4 M_{z}^{2}\right)\right]\right]\left(\frac{d y}{d z}\right)+\left[\frac{24}{r^{10}}(\mathbf{M} \mathbf{r})\left[3 x y M_{z}+3 y z M_{x}+5 x z M_{y}\right]-\right. \\
& \left.-\frac{360}{r^{12}}(\mathbf{M} \mathbf{r})^{2} x y z-\frac{24}{r^{8}}\left(x M_{z}+z M_{x}\right) M_{y}\right]\left(\frac{d x}{d z}\right)+\frac{180}{r^{12}}(\mathbf{M} \mathbf{r})^{2} y\left(x^{2}+y^{2}\right)+ \\
& +\frac{12}{r^{10}}(\mathbf{M} \mathbf{r})\left[\left(3 y^{2}-5 x^{2}\right) M_{y}-24(\mathbf{M} \mathbf{r}) y+y\left(8 x M_{x}+14 z M_{z}\right)\right]+ \\
& \left.+\frac{12}{r^{8}}\left[6(\mathbf{M} \mathbf{r}) M_{y}-y\left(2 M_{y}^{2}+M_{x}^{2}+M_{z}^{2}\right)-\left(x M_{x}+3 z M_{z}\right) M_{y}\right]\right\} .
\end{aligned}
$$

Equations (A1) and (A2) are non-linear, for which the conventional methods of solution [55] are not applicable. However, they contain the small parameter $\xi \mathbf{M}^{2} / r^{6}$. Therefore successive approximation is convenient method to solve these equations. For this, we substitute $x=x_{1,2}(z), y=y_{1,2}(z)$ and $t=t_{1,2}(z)$ In the form of expansions on the small parameter:

$$
\begin{gathered}
t_{1,2}(z)=T(z)+\eta_{1,2} \xi\left[\widetilde{t}(z)-\widetilde{t}\left(z_{s}\right)\right], \\
x_{1,2}(z)=X(z)+\eta_{1,2} \xi\left[\widetilde{x}(z)-\widetilde{x}\left(z_{d}\right)+\frac{\left(z_{d}-z\right)}{\left(z_{d}-z_{s}\right)}\left[\widetilde{x}\left(z_{d}\right)-\widetilde{x}\left(z_{s}\right)\right]\right], \\
y_{1,2}(z)=Y(z)+\eta_{1,2} \xi\left[\widetilde{y}(z)-\widetilde{y}\left(z_{s}\right)+\frac{\left(z_{d}-z\right)}{\left(z_{d}-z_{s}\right)}\left[\widetilde{y}\left(z_{d}\right)-\widetilde{y}\left(z_{s}\right)\right]\right],
\end{gathered}
$$


where $T(z), X(z), Y(z)$ are unknown functions of zero approximation, ănd $\widetilde{t}(z), \widetilde{x}(z), \widetilde{y}(z)$ are of the first approximation.

It is following from expressions (7), that initial conditions for the functions in these expressions have the form:

$$
T\left(z_{s}\right)=c t_{0}, X\left(z_{s}\right)=x_{s}, Y\left(z_{s}\right)=y_{s}, X\left(z_{d}\right)=x_{d}, Y\left(z_{d}\right)=y_{d} .
$$

In the Maxwellian approximation equations (A1)-(A2) give:

$$
\begin{gathered}
c^{2} \frac{d^{2} T(z)}{d z^{2}}=\frac{d^{2} X(z)}{d z^{2}}=\frac{d^{2} Y(z)}{d z^{2}}=0 \\
c^{2}\left(\frac{d T(z)}{d z}\right)^{2}-\left(\frac{d X(z)}{d z}\right)^{2}-\left(\frac{d Y(z)}{d z}\right)^{2}=1 .
\end{gathered}
$$

It is easy to obtain the solution of these equations satisfying the initial conditions ( $\breve{A} 4)$ :

$$
T(z)=t_{0}+\frac{N}{c}\left(z-z_{s}\right), \mathbf{R}=\mathbf{r}_{s}+\left(z-z_{s}\right) \mathbf{N},
$$

where notations

$$
\begin{gathered}
\mathbf{R}=\{X(z), Y(z), z\}, \quad N=|\mathbf{N}|, \\
N_{x}=\frac{\left(x_{d}-x_{s}\right)}{\left(z_{d}-z_{s}\right)}, N_{y}=\frac{\left(y_{d}-y_{s}\right)}{\left(z_{d}-z_{s}\right)}, N_{z}=1 .
\end{gathered}
$$

are used for the convenience of further calculations. With the use of expressions (A5) equations (A2) in the post-Maxvellian approximations have the form:

$$
\begin{aligned}
& \frac{d^{2} \widetilde{x}(z)}{d z^{2}}=\frac{1}{N^{12}}\left\{\left[\frac{180}{\rho^{12}}(\mathbf{M} \mathbf{R})^{2} X^{2} z+\frac{12 N^{2}}{\rho^{10}}(\mathbf{M} \mathbf{R})\left[9(\mathbf{M} \mathbf{R}) z-z\left(14 X M_{x}+8 Y M_{y}\right)+\quad(A 7)\right.\right.\right. \\
+ & \left.\left.\left(3 X^{2}+8 Y^{2}\right) M_{z}\right]+\frac{12 N^{4}}{\rho^{8}}\left[z\left(\mathbf{M}^{2}+M_{z}^{2}\right)-9(\mathbf{M} \mathbf{R}) M_{z}+\left(3 X M_{x}+Y M_{y}\right) M_{z}\right]\right] N_{x}^{3}+\left[\frac{360}{\rho^{12}}(\mathbf{M} \mathbf{R})^{2} X Y z+\right. \\
+ & \left.\frac{24 N^{4}}{\rho^{8}} M_{z}\left[X M_{y}+Y M_{x}\right]-\frac{24 N^{2}}{\rho^{10}}(\mathbf{M} \mathbf{R})\left[3 Y z M_{x}+3 X z M_{y}+5 X Y M_{z}\right]\right] N_{x}^{2} N_{y}+\left[\frac{180}{\rho^{12}}(\mathbf{M ~ R})^{2} Y^{2} z+\right. \\
+ & \frac{12 N^{2}}{\rho^{10}}(\mathbf{M} \mathbf{R})\left[9(\mathbf{M} \mathbf{R}) z+\left(8 X^{2}+3 Y^{2}\right) M_{z}-z\left(8 X M_{x}+14 Y M_{y}\right)\right]+\frac{12 N^{4}}{\rho^{8}}\left[z\left(\mathbf{M}^{2}+M_{z}^{2}\right)+\right. \\
+ & \left.\left.\left(X M_{x}+3 Y M_{y}\right) M_{z}-9(\mathbf{M} \mathbf{R}) M_{z}\right]\right] N_{x} N_{y}^{2}+\left[\frac { 2 4 N ^ { 2 } } { \rho ^ { 1 0 } } ( \mathbf { M } \mathbf { R } ) \left[15(\mathbf{M} \mathbf{R}) Y+3\left(2 X^{2}+Y^{2}\right) M_{y}+\right.\right. \\
+ & \left.8 Y\left(X M_{x}-z M_{z}\right)\right]-\frac{360}{\rho^{12}}(\mathbf{M} \mathbf{R})^{2} Y\left(2 X^{2}+Y^{2}\right)+\frac{24 N^{4}}{\rho^{8}}\left[\left(z M_{z}-X M_{x}\right) M_{y}-3(\mathbf{M} \mathbf{R}) M_{y}+\right. \\
+ & \left.\left.Y\left(M_{z}^{2}-M_{x}^{2}\right)\right]\right] N_{x} N_{y}+\left[\frac{12 N^{2}}{\rho^{10}}(\mathbf{M} \mathbf{R})\left[21(\mathbf{M} \mathbf{R}) X+25 X^{2} M_{x}+6 Y^{2} M_{x}+8 X\left(Y M_{y}-z M_{z}\right)\right]-\right. \\
& \left.\frac{180}{\rho^{12}}(\mathbf{M} \mathbf{R})^{2} X\left(3 X^{2}+2 Y^{2}\right)-\frac{12 N^{4}}{\rho^{8}}\left[5(\mathbf{M} \mathbf{R}) M_{x}+X\left(4 M_{x}^{2}+M_{y}^{2}-M_{z}^{2}\right)+\left(Y M_{y}-z M_{z}\right) M_{x}\right]\right] N_{x}^{2}+ \\
+ & {\left[\frac{12 N^{2}}{\rho^{10}}(\mathbf{M} \mathbf{R})\left[\left(8 X^{2}+5 Y^{2}\right) M_{x}-9(\mathbf{M} \mathbf{R}) X+X\left(14 Y M_{y}+8 z M_{z}\right)\right]-\frac{180}{\rho^{12}}(\mathbf{M} \mathbf{R})^{2} X Y^{2}+\right.}
\end{aligned}
$$




$$
\begin{gathered}
\left.+\frac{12 N^{4}}{\rho^{8}}\left[(\mathbf{M} \mathbf{R}) M_{x}-X\left(2 M_{x}^{2}+M_{y}^{2}+M_{z}^{2}\right)-\left(3 Y M_{y}+z M_{z}\right) M_{x}\right]\right] N_{y}^{2}+\left[\frac { 1 2 N ^ { 2 } } { \rho ^ { 1 0 } } ( \mathbf { M } \mathbf { R } ) \left[24 z(\mathbf{M} \mathbf{R})+\left(25 X^{2}+\right.\right.\right. \\
\left.\left.+19 Y^{2}\right) M_{z}+8 z\left(X M_{x}-Y M_{y}\right)\right]-\frac{180}{\rho^{12}}(\mathbf{M} \mathbf{R})^{2} z\left(3 X^{2}+Y^{2}\right)-\frac{12 N^{4}}{\rho^{8}}\left[20(\mathbf{M} \mathbf{R}) M_{z}+\right. \\
\left.\left.+\left(X M_{x}-Y M_{y}\right) M_{z}+z\left(M_{x}^{2}-M_{y}^{2}-4 M_{z}^{2}\right)\right]\right] N_{x}+\left[\frac{24 N^{2}}{\rho^{10}}(\mathbf{M} \mathbf{R})\left[3 X Y M_{z}+3 X z M_{y}+5 Y z M_{x}\right]-\right. \\
\left.-\frac{360}{\rho^{12}}(\mathbf{M} \mathbf{R})^{2} X Y z-\frac{24 N^{4}}{\rho^{8}}\left(Y M_{z}+z M_{y}\right) M_{x}\right] N_{y}+\frac{180}{\rho^{12}}(\mathbf{M ~ R})^{2} X\left(X^{2}+Y^{2}\right)+ \\
+\frac{12 N^{2}}{\rho^{10}}(\mathbf{M} \mathbf{R})\left[\left(3 X^{2}-5 Y^{2}\right) M_{x}-24(\mathbf{M} \mathbf{R}) X+X\left(8 Y M_{y}+14 z M_{z}\right)\right]+ \\
\left.+\frac{12 N^{4}}{\rho^{8}}\left[6(\mathbf{M ~ R}) M_{x}-X\left(2 M_{x}^{2}+M_{y}^{2}+M_{z}^{2}\right)-\left(Y M_{y}+3 z M_{z}\right) M_{x}\right]\right\}
\end{gathered}
$$

$\frac{d^{2} \widetilde{y}(z)}{d z^{2}}=\frac{1}{N^{12}}\left\{\left[\frac{180}{\rho^{12}}(\mathbf{M} \mathbf{R})^{2} Y^{2} z+\frac{12 N^{2}}{\rho^{10}}(\mathbf{M} \mathbf{R})\left[9(\mathbf{M ~ R}) z-z\left(14 Y M_{y}+8 X M_{x}\right)+\left(3 Y^{2}+8 X^{2}\right) M_{z}\right]+\right.\right.$ $\left.+\frac{12 N^{4}}{\rho^{8}}\left[z\left(\mathbf{M}^{2}+M_{z}^{2}\right)-9(\mathbf{M} \mathbf{R}) M_{z}+\left(3 Y M_{y}+X M_{x}\right) M_{z}\right]\right] N_{y}^{3}+\left[\frac{360}{\rho^{12}}(\mathbf{M} \mathbf{R})^{2} X Y z+\right.$ $\left.+\frac{24 N^{4}}{\rho^{8}} M_{z}\left[X M_{y}+Y M_{x}\right]-\frac{24 N^{2}}{\rho^{10}}(\mathbf{M} \mathbf{R})\left[3 z\left(X M_{y}+Y M_{x}\right)+5 X Y M_{z}\right]\right] N_{y}^{2} N_{x}+$ $+\left[\frac{180}{\rho^{12}}(\mathbf{M ~ R})^{2} X^{2} z+\frac{12 N^{2}}{\rho^{10}}(\mathbf{M ~ R})\left[9(\mathbf{M ~ R}) z+\left(8 Y^{2}+3 X^{2}\right) M_{z}-z\left(8 Y M_{y}+14 X M_{x}\right)\right]+\right.$ $\left.+\frac{12 N^{4}}{\rho^{8}}\left[z\left(\mathbf{M}^{2}+M_{z}^{2}\right)+\left(Y M_{y}+3 X M_{x}\right) M_{z}-9(\mathbf{M} \mathbf{R}) M_{z}\right]\right] N_{y} N_{x}^{2}+\left[\frac{24 N^{2}}{\rho^{10}}(\mathbf{M} \mathbf{R})[15(\mathbf{M} \mathbf{R}) X+\right.$ $\left.+3\left(2 Y^{2}+X^{2}\right) M_{x}+8 X\left(Y M_{y}-z M_{z}\right)\right]-\frac{360}{\rho^{12}}(\mathbf{M ~ R})^{2} X\left(2 Y^{2}+X^{2}\right)+\frac{24 N^{4}}{\rho^{8}}\left[\left(z M_{z}-Y M_{y}\right) M_{x}-\right.$ $\left.\left.-3(\mathbf{M} \mathbf{R}) M_{x}+X\left(M_{z}^{2}-M_{y}^{2}\right)\right]\right] N_{y} N_{x}+\left[\frac{12 N^{2}}{\rho^{10}}(\mathbf{M ~ R})\left[21(\mathbf{M ~ R}) Y+25 Y^{2} M_{y}+6 X^{2} M_{y}+8 Y\left(X M_{x}-\right.\right.\right.$ $\left.\left.-z M_{z}\right)\right]-\frac{180}{\rho^{12}}(\mathbf{M ~ R})^{2} Y\left(3 Y^{2}+2 X^{2}\right)-\frac{12 N^{4}}{\rho^{8}}\left[5(\mathbf{M ~ R}) M_{y}+Y\left(4 M_{y}^{2}+M_{x}^{2}-M_{z}^{2}\right)+\right.$ $\left.\left.+\left(X M_{x}-z M_{z}\right) M_{y}\right]\right] N_{y}^{2}+\left[\frac{12 N^{2}}{\rho^{10}}(\mathbf{M} \mathbf{R})\left[\left(8 Y^{2}+5 X^{2}\right) M_{y}-9(\mathbf{M} \mathbf{R}) Y+Y\left(14 X M_{x}+\right.\right.\right.$ $\left.\left.\left.+8 z M_{z}\right)\right]-\frac{180}{\rho^{12}}(\mathbf{M} \mathbf{R})^{2} Y X^{2}+\frac{12 N^{4}}{\rho^{8}}\left[(\mathbf{M} \mathbf{R}) M_{y}-Y\left(2 M_{y}^{2}+M_{x}^{2}+M_{z}^{2}\right)-\left(3 X M_{x}+z M_{z}\right) M_{y}\right]\right] N_{x}^{2}+$ $+\left[\frac{12 N^{2}}{\rho^{10}}(\mathbf{M} \mathbf{R})\left[24 z(\mathbf{M} \mathbf{R})+\left(25 Y^{2}+19 X^{2}\right) M_{z}+8 z\left(Y M_{y}-X M_{x}\right)\right]-\frac{180}{\rho^{12}}(\mathbf{M ~ R})^{2} z\left(3 Y^{2}+X^{2}\right)-\right.$ $\left.-\frac{12 N^{4}}{\rho^{8}}\left[20(\mathbf{M} \mathbf{R}) M_{z}+\left(Y M_{y}-X M_{x}\right) M_{z}+z\left(M_{y}^{2}-M_{x}^{2}-4 M_{z}^{2}\right)\right]\right] N_{y}+\left[\frac{24 N^{2}}{\rho^{10}}(\mathbf{M ~ R}) \times\right.$ $\left.\times\left[3 X Y M_{z}+3 Y z M_{x}+5 X z M_{y}\right]-\frac{360}{\rho^{12}}(\mathbf{M} \mathbf{R})^{2} X Y z-\frac{24 N^{4}}{\rho^{8}}\left(X M_{z}+z M_{x}\right) M_{y}\right] N_{x}+$ 


$$
\begin{gathered}
+\frac{180}{\rho^{12}}(\mathbf{M} \mathbf{R})^{2} Y\left(X^{2}+Y^{2}\right)+\frac{12 N^{2}}{\rho^{10}}(\mathbf{M} \mathbf{R})\left[\left(3 Y^{2}-5 X^{2}\right) M_{y}-24(\mathbf{M} \mathbf{R}) Y+Y\left(8 X M_{x}+14 z M_{z}\right)\right]+ \\
\left.+\frac{12 N^{4}}{\rho^{8}}\left[6(\mathbf{M} \mathbf{R}) M_{y}-Y\left(2 M_{y}^{2}+M_{x}^{2}+M_{z}^{2}\right)-\left(X M_{x}+3 z M_{z}\right) M_{y}\right]\right\}
\end{gathered}
$$

where

$$
\begin{gathered}
\rho=\sqrt{(z+p)^{2}+q^{2}}, \quad X=x_{s}+N_{x}\left(z-z_{s}\right), Y=y_{s}+N_{y}\left(z-z_{s}\right), \\
p=\frac{\left[\left(x_{d}-x_{s}\right)\left(x_{s} z_{d}-z_{s} x_{d}\right)+\left(y_{d}-y_{s}\right)\left(y_{s} z_{d}-z_{s} y_{d}\right)\right]}{\left(\mathbf{r}_{d}-\mathbf{r}_{\mathbf{s}}\right)^{2}}, \\
q^{2}=\frac{\left(z_{d}-z_{s}\right)^{2}}{\left(\mathbf{r}_{d}-\mathbf{r}_{\mathbf{s}}\right)^{4}}\left[\left(x_{s} y_{d}-y_{s} x_{d}\right)^{2}+\left(x_{s} z_{d}-z_{s} x_{d}\right)^{2}+\left(y_{s} z_{d}-z_{s} y_{d}\right)^{2}\right] .
\end{gathered}
$$

The first integral (A1) in this approximation gives the equation for determination of the function $\widetilde{t}(z)$ :

$$
\begin{gathered}
c N \frac{d \widetilde{t}(z)}{d z}-2\left[N_{x}^{2}+N_{y}^{2}+1\right]\left[\frac{3(\mathbf{M} \mathbf{R})^{2}}{N^{8} \rho^{8}}+\frac{\mathbf{M}^{2}}{N^{6} \rho^{6}}\right]- \\
-\left[N_{x} \frac{d \widetilde{x}(z)}{d z}+N_{y} \frac{d \widetilde{y}(z)}{d z}\right]+\frac{2}{N^{6} \rho^{6}}\left[\frac{3(\mathbf{M} \mathbf{R})}{N^{2} \rho^{2}}(\mathbf{R} \mathbf{N})-(\mathbf{M} \mathbf{N})\right]^{2}=0 .
\end{gathered}
$$

Before to integrate the equations (A7) and (A9), we make some estimations. According to the statement of the problem, the source of hard emission is on the neutron star surface or in it magnetosphere. Taking the neutron star radius equal to the $10 \mathrm{~km}$, we can assume that $\sqrt{x_{s}^{2}+y_{s}^{2}+z_{s}^{2}}<10^{2} \mathrm{~km}$. Detector of hard emission is on the near-Earth satellite. Usually, two types of orbits are appropriate for such observations. First one is the lowaltitude circular orbit, which lays mainly under the Earth radiation belts. Its radius no more than approximately $7 \cdot 10^{3} \mathrm{~km}$. The other type of an orbit is high-apogee elliptical one, on which satellite mainly is out of the Earth magnetosphere. The apogee altitude for such orbits (INEGRAL space observatory for ex.) can be about $10^{5}$ łn̆. Therefore, we can use approximations $\left|x_{d}\right| \leq 10^{5} \mathrm{~km}$ and $\left|y_{d}\right| \leq 10^{5} \mathrm{~km}$ for coordinates $x_{d}$ I $y_{d}$.

The coordinate $z_{d}$ value in the chosen coordinate system is the same as the distance from the pulsar to the Earth. Because the nearest pulsar is on the distance of about a few kps, i.e. $\sim 10^{17} \mathrm{~km}$ from the Earth, we assume that $z_{d} \sim 10^{17} \mathrm{~km}$. Substituting these values in the expressions (A6), we obtain the following estimations: $N_{x} \sim N_{y} \sim 10^{-12}, N_{z}=1$. Therefore, we can simplify significantly the equations (A7) and (A9), keep there only asymptotically main terms in the expansion on the small parameters $N_{x} 1 N_{y} \sim 10^{-12}$.

In this case it is following from equations (A5) and (A8), that $\mathbf{R}=\left\{x_{s}, y_{s}, z\right\}, X=$ $x_{s}, Y=y_{s}, N_{x}=N_{y}=0, N=1, p=0, q^{2}=x_{s}^{2}+y_{s}^{2}, \rho=\sqrt{z^{2}+q^{2}}$. Thus, equations (A7) have the form:

$$
\begin{gathered}
\frac{d^{2} \widetilde{x}(z)}{d z^{2}}=\frac{180}{\rho^{12}}(\mathbf{M} \mathbf{R})^{2} x_{s}\left(x_{s}^{2}+y_{s}^{2}\right)+\frac{12}{\rho^{10}}(\mathbf{M} \mathbf{R})\left[\left(3 x_{s}^{2}-5 y_{s}^{2}\right) M_{x}-24(\mathbf{M} \mathbf{R}) x_{s}+\right. \\
\left.+x_{s}\left(8 y_{s} M_{y}+14 z M_{z}\right)\right]+\frac{12}{\rho^{8}}\left[6(\mathbf{M} \mathbf{R}) M_{x}-x_{s}\left(2 M_{x}^{2}+M_{y}^{2}+M_{z}^{2}\right)-\left(y_{s} M_{y}+3 z M_{z}\right) M_{x}\right] \\
\frac{d^{2} \widetilde{y}(z)}{d z^{2}}=\frac{180}{\rho^{12}}(\mathbf{M ~ R})^{2} y_{s}\left(x_{s}^{2}+y_{s}^{2}\right)+\frac{12}{\rho^{10}}(\mathbf{M} \mathbf{R})\left[\left(3 y_{s}^{2}-5 x_{s}^{2}\right) M_{y}-24(\mathbf{M ~ R}) y_{s}+\right.
\end{gathered}
$$




$$
\left.+y_{s}\left(8 x_{s} M_{x}+14 z M_{z}\right)\right]+\frac{12}{\rho^{8}}\left[6(\mathbf{M ~ R}) M_{y}-y_{s}\left(2 M_{y}^{2}+M_{x}^{2}+M_{z}^{2}\right)-\left(x_{s} M_{x}+3 z M_{z}\right) M_{y}\right] .
$$

The first integral (A9) also becomes more simple in this case

$$
c \frac{d \widetilde{t}(z)}{d z}-2\left[\frac{3(\mathbf{M} \mathbf{R})^{2}}{\rho^{8}}+\frac{\mathbf{M}^{2}}{\rho^{6}}\right]+\frac{2}{\rho^{6}}\left[\frac{3(\mathbf{M} \mathbf{R})}{\rho^{2}} z-M_{z}\right]^{2}=0 .
$$

To solve equations (A10), we obtain:

$$
\begin{aligned}
& \widetilde{x}(z)=\frac{3}{64 q^{9}} \operatorname{atan}\left(\frac{z}{q}\right)\left[2 M_{x} M_{z} q^{4}-175 z x_{s}\left(M_{x} x_{s}+M_{y} y_{s}\right)^{2}+\right. \\
& +10 q^{2}\left(5 M_{z} x_{s}-3 z M_{x}\right)\left(M_{x} x_{s}+M_{y} y_{s}\right)-5 z x_{s} q^{2}\left(16 M_{y}^{2}+15 M_{z}^{2}\right)+ \\
& \left.+80 y_{s} z q^{2} M_{x} M_{y}\right]+\frac{1}{64 \rho^{2} q^{6}}\left[30\left(5 x_{s} z M_{z}+M_{x} q^{2}\right)\left(M_{x} x_{s}+M_{y} y_{s}\right)+\right. \\
& +2 q^{2} M_{x}\left(3 M_{z} z-40 M_{y} y_{s}\right)+5 x_{s} q^{2}\left(16 M_{y}^{2}+15 M_{z}^{2}\right)+175 x_{s}\left(M_{x} x_{s}+\right. \\
& \left.\left.+M_{y} y_{s}\right)^{2}\right]+\frac{1}{32 \rho^{4} q^{4}}\left[x_{s} q^{2}\left(16 M_{y}^{2}+15 M_{z}^{2}\right)+\left(6 M_{x} q^{2}+50 x_{s} z M_{z}\right) \times\right. \\
& \left.\times\left(M_{x} x_{s}+M_{y} y_{s}\right)+2 q^{2} M_{x}\left(M_{z} z-8 M_{y} y_{s}\right)+35 x_{s}\left(M_{x} x_{s}+M_{y} y_{s}\right)^{2}\right]+ \\
& +\frac{5}{8 \rho^{6} q^{2}}\left[x_{s}\left(M_{x} x_{s}+M_{y} y_{s}\right)^{2}+q^{2}\left(2 M_{z} M_{x} z-M_{z}^{2} x_{s}\right)+2\left(M_{x} q^{2}+\right.\right. \\
& \left.\left.+M_{z} x_{s} z\right)\left(M_{x} x_{s}+M_{y} y_{s}\right)\right]-\frac{9 x_{s}(\mathbf{M} \mathbf{R})^{2}}{4 \rho^{8}}, \\
& \widetilde{y}(z)=\frac{3}{64 q^{9}} \operatorname{atan}\left(\frac{z}{q}\right)\left[2 M_{y} M_{z} q^{4}-175 z y_{s}\left(M_{x} x_{s}+M_{y} y_{s}\right)^{2}+\right. \\
& +10 q^{2}\left(5 M_{z} y_{s}-3 M_{y} z\right)\left(M_{x} x_{s}+M_{y} y_{s}\right)-5 z y_{s} q^{2}\left(16 M_{x}^{2}+15 M_{z}^{2}\right)+ \\
& \left.+80 x_{s} z q^{2} M_{x} M_{y}\right]+\frac{1}{64 \rho^{2} q^{6}}\left[30\left(5 M_{z} y_{s} z+M_{y} q^{2}\right)\left(M_{x} x_{s}+M_{y} y_{s}\right)-\right. \\
& +2 q^{2} M_{y}\left(3 M_{z} z-40 M_{x} x_{s}\right)+5 y_{s} q^{2}\left(16 M_{x}^{2}+15 M_{z}^{2}\right)+175 y_{s}\left(M_{x} x_{s}+\right. \\
& \left.\left.+M_{y} y_{s}\right)^{2}\right]+\frac{1}{32 \rho^{4} q^{4}}\left[y_{s} q^{2}\left(16 M_{x}^{2}+15 M_{z}^{2}\right)+\left(6 M_{y} q^{2}+50 M_{z} y_{s} z\right) \times\right. \\
& \left.\times\left(M_{x} x_{s}+M_{y} y_{s}\right)+2 q^{2} M_{y}\left(M_{z} z-8 M_{x} x_{s}\right)+35 y_{s}\left(M_{x} x_{s}+M_{y} y_{s}\right)^{2}\right]+ \\
& +\frac{5}{8 \rho^{6} q^{2}}\left[y_{s}\left(M_{x} x_{s}+M_{y} y_{s}\right)^{2}+q^{2}\left(2 M_{z} M_{y} z-M_{z}^{2} y_{s}\right)+\right. \\
& \left.+2\left(M_{y} q^{2}+M_{z} y_{s} z\right)\left(M_{x} x_{s}+M_{y} y_{s}\right)\right]-\frac{9 y_{s}(\mathbf{M} \mathbf{R})^{2}}{4 \rho^{8}} .
\end{aligned}
$$

Integrating the equation (A11), we find the explicit dependence $\widetilde{t}(z)$, i.e. the law of a hard emission pulse move along beams from it common source to the detector:

$$
\widetilde{t}(z)=\left[25\left(M_{x} x_{s}+M_{y} y_{s}\right)^{2}+q^{2}\left(16 \mathbf{M}^{2}-M_{z}^{2}\right)\right]\left[\frac{z\left(3 \rho^{2}+2 q^{2}\right)}{64 \rho^{4} q^{6}}+\frac{3}{64 q^{7}} \operatorname{atan}\left(\frac{z}{q}\right)\right]+
$$




$$
\begin{aligned}
+\frac{1}{8 \rho^{6} q^{2}}\left[5 z\left(M_{x} x_{s}+M_{y} y_{s}\right)^{2}+\right. & \left.16 q^{2} M_{z}\left(M_{x} x_{s}+M_{y} y_{s}\right)+3 M_{z}^{2} q^{2} z\right]+\frac{9}{4 \rho^{8}}\left[z\left(M_{x} x_{s}+M_{y} y_{s}\right)^{2}-\right. \\
& \left.-q^{2} M_{z}\left(2 M_{x} x_{s}+2 M_{y} y_{s}+M_{z} z\right)\right] .
\end{aligned}
$$

It is necessary to note, that expressions (A12) and (A13) are the partial solutions of non-uniform equations (A10) and (A11). From the mathematics rules, they should be added by the general solutions of corresponding uniform equations, i.e. make replacement

$$
\widetilde{t}(z) \rightarrow \widetilde{t}(z)+a_{0}, \quad \widetilde{x}(z) \rightarrow \widetilde{x}(z)+a_{1}+b_{1} z, \quad \widetilde{y}(z) \rightarrow \widetilde{y}(z)+a_{2}+b_{2} z,
$$

where $a_{0}, a_{1}, a_{2}, b_{1}, b_{2}$ are the arbitrary constants.

However, if to substitute these general solutions (A10) and (A11) in the expressions (A3) then all constants $a_{0}, a_{1}, a_{2}, b_{1}, b_{2}$ exactly cancel. Therefore, further we will use more simple expressions (A12) and (A13).

\section{References}

[1] Tindo I.P., et. al., Solar Phys., 24 (1972) 429.

[2] Denisov Yu.I. et. al., Astron. vestnik, 37 (2003) 127.

[3] (http://hesperia.gsfc.nasa.gov/hessi/).

[4] Weisskopf M. C. et al. A precision measurement of the X-ray polarization of the Crab Nebula without pulsar contamination, Astrophysical Journal 220 (1978) L117.

[5] Lei F. et al., Characteristics of COMPTEL as a polarimeter and its data analysis, Astronomy and Astrophysics Supplement 120 (1996) 695.

[6] Sidoli L., Paizis A. and Postnov K. INTEGRAL study of temporal properties of bright flares in Supergiant Fast X-ray Transients, Monthly Notices of the Royal Astronomical Society $\mathbf{4 5 7}$ (2016) 3693.

[7] Laurent P. et.al. Polarized Gamma-Ray Emission from the Galactic Black Hole Cygnus X-1, Science 332 (2011) 438.

[8] Götz Diego et.al. Variable Polarization Measured in the Prompt Emission of GRB 041219A Using IBIS on Board INTEGRAL, ApJ 695 (2009) 208.

[9] Chauvin M. et. al. Polarimetry in the Hard X-Ray Domain with INTEGRAL SPI, ApJ, 769 (2013) 137.

[10] Rodriguez J. Spectral State Dependence of the 0.4-2 MeV Polarized Emission in Cygnus X-1 Seen with INTEGRAL/IBIS, and Links with the AMI Radio Data, ApJ 807 (2015) 17.

[11] Forot M. et.al. Polarization of the Crab Pulsar and Nebula as Observed by the INTEGRAL/IBIS Telescope, Astrophysical Journal Letters 688 (2008) L29 .

[12] Chauvin M. et al., Observation of polarized hard X-ray emission from the Crab by the PoGOLite Pathfinder, MNRAS 456 (2016) 84.

[13] Mizuno T. et al., Beam test of a prototype detector array for the PoGO astronomical hard x-ray / soft gamma-ray polarimeter, Nucl. Instrum. Meth. A540 (2005) 158.

[14] Bloser P.F. et al., Calibration of the Gamma-Ray Polarimeter Experiment (GRAPE) at a polarized hard X-ray beam, Nucl. Instrum. Meth. A600 (2009) 424.

[15] Orsi S. et al., Response of the Compton polarimeter POLAR to polarized hard X-rays, Nucl. Instrum. Meth. A648 (2011) 139. 
[16] Zoglauer A. et al., Polarization Measurements with the MEGA Telescope, Proceedings of the 5th INTEGRAL Workshop on the INTEGRAL Universe (ESA SP-552), 16-20 February 2004, Munich, Germany.

[17] http://astrogam.iaps.inaf.it/

[18] Greiner J. et al., GRIPS - Gamma-Ray Imaging, Polarimetry and Spectroscopy, Exper.Astron. 34 (2012) 551.

[19] O'Neill T.J. et al., Tracking, imaging and polarimeter properties of the TIGRE instrument, Astronomy and Astrophysics Supplement, 120 (1996) 661.

[20] Caroli E. et al., A CdTe position sensitive spectrometer for hard X-and soft -ray polarimetry, Nucl. Instrum. Meth. A477 (2002) 567.

[21] Aprile E. et al., Compton Imaging of MeV Gamma-Rays with the Liquid Xenon Gamma-Ray Imaging Telescope (LXeGRIT), Nucl. Instrum. Meth. A593 (2008) 414.

[22] Tanimori T. et al., An Electron-Tracking Compton Telescope for a Survey of the Deep Universe by $\mathrm{MeV}$ gamma-rays, ApJ, $\mathbf{8 1 0}$ (2015) 28.

[23] Denisov D.M., Effects of nonlinear electrodynamics in the magnetic field of a pulsar, Canadian Journal of Physics, 92 (2014) 1453.

[24] Abishev M. et.al., Some astrophysical effects of nonlinear vacuum electrodynamics in the magnetosphere of a pulsar, Astroparticle Physics 73 (2016) 8.

[25] Denisov V.I. and Svertilov S.I., Nonlinear electromagnetic and gravitational actions of neutron star fields on electromagnetic wave propagation, Phys.Rev. D71 (2005) 063002.

[26] Heyl, J., S., Shaviv, N., J., Mon. Not. R. Astron. Soc 311 (2000) 555.

[27] Mignani R. P. et. al., Evidence for vacuum birefringence from the first optical polarimetry measurement of the isolated neutron star RX J1856.5-3754, Mon. Not. R. Astron. Soc 465 (2017) 492.

[28] Heisenberg W. and Euler H., Consequences of Dirac's Theory of Positrons, Z. Phys. 98 (1936) 714 .

[29] Born M. and Infeld L., Foundations of the New Field Theory, Proc. Roy. Soc. A144 (1934) 425.

[30] Grib A.A., Mamaev S.G. and Mostepanenko V.M., Kvantovye effekty v intensivnyh vneshnih polyah, (1988) Energoatomizdat, Moscow.

[31] Adler S.L., Photon splitting and photon dispersion in a strong magnetic field, Ann. Phys. (N.Y.) 67 (1971) 599.

[32] Meszaros P., High Energy Radiation from Magnetized Neutron Stars, Univ. of Chicago Press, Chicago, (1992).

[33] Ventura J., Nagel W. and P. Meszaros, Possible vacuum signature in the spectra of X-ray pulsars, Astrophys. J. (Letters), 233 (1979) L125.

[34] Gnedin Yu.N., Effect of vacuum polarization in a strong magnetic field and spectral features of $X$-ray source emission, in High resolution X-ray spectroscopy of cosmic plasmas, Cambridge, England and New York, Cambridge University Press (1990) 78.

[35] Gal'tsov D.V. and Nikitina N.S., Macroscopic vacuum effects in an inhomogeneous and nonstationary electromagnetic field, JETP 57 (1983) 705.

[36] Bussard R.W., Alexander S.B. and Meszaros P., One- and two-photon scattering in strong magnetic fields, Phys. Rev. D34 (1986) 440.

[37] Denisov V.I. et.al. Phys. Rev. D94 (2016) 045021.

[38] Denisov V.I. et.al. Eur. Phys. J. C76 (2016) 612. 
[39] de Lorenci V.A. et.al. Dyadosphere bending of light, Astron. \& Astrophys., 369 (2001) 690.

[40] Denisov V.I. and Svertilov S.I., Vacuum nonlinear electrodynamics curvature of photon trajectories in pulsars and magnetars, Astronomy \& Astrophysics, 399 (2003) L39.

[41] Denisov V.I., Denisova I. P. and Sokolov V.A., Theoretical and Mathematical Physics, 172 , (2012) 1321.

[42] Riffert H. and Meszaros P., Gravitational light bending near neutron stars. I - Emission from columns and hot spots, Astrophys. J. 325 (1988) 207.

[43] Meszaros P. and Riffert H., Gravitational light bending near neutron stars. II - Accreting pulsar spectra as a function of phase, Astrophys. J. 327 (1988) 712.

[44] Gapochka M.G. et al., Computational Mathematics and Mathematical Physics, 55 (2015) 1857.

[45] Denisov V.I., Sokolov V.A. and Vasili'ev M.I., Phys. Rev. D90 (2014) 02301.

[46] Silant'ev N.A., Gnedin Yu.N. and Krymski T.Sh., Faraday rotation and polarization of light scattered in magnetized stellar wind, Astron. \& Astrophys., 357 (2000) 1151.

[47] Gnedin Yu.N., Silant'ev N.A. and Shternin P.S., Polarization of radiation from a strongly magnetized accretion disk: The asymptotoc spectral distribution, Astron. Letters 32 (2006) 39.

[48] Gnedin Yu.N., Silant'ev N.A. and Piotrovich M.Yu., Polarization of the radiation scattered in conical magnetized envelope, Astron. Letters 32 (2006) 96.

[49] Lifshitz E.M., Pitaevskii L.P., Physical Kinetics. Vol. 10, (1st ed.), (1981), Pergamon Press.

[50] Gnedin Yu.N. et.al. The polarization effects of radiation from magnetized envelopes and accretion structures, astro-ph/0406288, (2004).

[51] Gnedin Yu.N. and Krasnikov S.V., Polarimetric effects associated with the detection of Goldstone bosons in stars and galaxies, JETP 75 (1992) 933.

[52] Bennett K., et al., COS-B observations of pulsed gamma-ray emission from PSR 0531 plus 21 and PSR 0833-45, Astron. \& Astrophys. 61 (1977) 279.

[53] Natalucci L. et al., X/gamma-ray observation of the Crab pulsar with the Sigma telescope, Adv. Space Res. 11 (1991) 79.

[54] Bogomolov A.V., et.al, The properties of gamma-radiation and high energy neutron fluxes in "MIR" station orbit, Radiation Measurements 35 (2002) 473.

[55] Zhukovsky K.V., Operational solution for some types of second order differential equations and for relevant physical problems, Journal of Mathematical Analysis and Applications 446 (2017) 628. 